\title{
Information geometry encoded in bulk geometry
}

\author{
Asato Tsuchiya and Kazushi Yamashiro \\ Department of Physics, Shizuoka University, \\ 836 Ohya, Suruga-ku, Shizuoka 422-8529, Japan \\ Graduate School of Science and Technology, Shizuoka University, \\ 3-5-1 Johoku, Naka-ku, Hamamatsu 432-8011, Japan \\ E-mail: tsuchiya.asato@shizuoka.ac.jp, \\ yamashiro.kazushi.17@shizuoka.ac.jp
}

ABSTRACT: We study how information geometry is described by bulk geometry in the gauge/gravity correspondence. We consider a quantum information metric that measures the distance between the ground states of a CFT and a theory obtained by perturbing the CFT. We find a universal formula that represents the quantum information metric in terms of back reaction to the AdS bulk geometry.

KEYwords: AdS-CFT Correspondence, Gauge-gravity correspondence

ARXiv ePrint: 2002.11365 


\section{Contents}

1 Introduction 1

2 Information metric in field theory 2

3 Information metric as on-shell action 4

4 Back reaction to the AdS geometry 5

5 Vector field $\quad 9$

6 Tensor field 11

7 Conclusion and discussion $\quad 13$

$\begin{array}{ll}\text { A Ricci tensor and scalar curvature } & 14\end{array}$

\section{Introduction}

Emergence of space-time (geometry) is considered to play an essential role in constructing quantum theory of gravity. Indeed, it is observed in various contexts including the AdS/CFT correspondence or the gauge/gravity correspondence [1-3], where the bulk direction on the gravity side emerges as the scale of renormalization group on the field theory side $[2-5]$. This observation motivates one to reconstruct full bulk geometry from field theory. The Ryu-Takayanagi formula [6] gives a hint to this problem. It relates entanglement entropy of a region in space on which a field theory is defined to the area of a minimal surface in the bulk whose boundary agrees with that of the region. Thus, it gives a relationship between quantum information theory and bulk geometry.

In this paper, to further gain insights into this problem, we consider information metric in quantum information theory other than entanglement entropy, and investigate how they are encoded in bulk geometry. We represent information metric in terms of back reaction to the AdS bulk geometry, which is determined by dynamics of gravity. The geometrical quantity associated with the information metric is local in the bulk direction, while the minimal surface associated with entanglement entropy is not. Information metrics have been studied in the context of the AdS/CFT correspondence in [7-15].

The authors of [7] considered a CFT and a theory that is obtained by perturbing the CFT by an operator and calculate an information metric that measures the distance between the ground states of these two theories. They examined a gravity dual of a filed theory that is obtained by gluing the above two theories and found that the information metric is represented by the volume of a hypersurface that is the time slice in the bulk 
wihich ends on the time slice on the boundary. Further developments in this derection have been made in [8-11]. A different type of information metric has been investigated in [13-15].

We consider the same set of two filed theories and the same information metric as those in [7]. Then, we examine two gravity duals, one of which is dual to the CFT and the other of which is dual to the perturbed CFT. The bulk geometry in the latter gravity dual gains the back reaction caused by the perturbation. We find a formula (4.23) that expresses the information metric by deviation of the volume of a hypersurface in the bulk from that in the case of the AdS geometry. This formula is universal in the sense that it holds for each case in which the perturbation is given by a scalar, vector or tensor operator. The formula is new findings in this paper.

This paper is organized as follows. In section 2, we briefly review information metrics in field theories. In section 3, we consider a CFT and a theory that is obtained by perturbing the CFT by a scalar primary operator. We calculate the information metric that measures the distance between the ground states of these two theories, and associate it to the on-shell action on the gravity side by using the GKP-Witten relation. In section 4, we evaluate the back reaction to the AdS geometry caused by the perturbation and find the formula that relates the information metric to deviation of the volume of the hypersurface in the bulk from that in the case of the AdS geometry. In sections 5 and 6, we examine the cases in which CFTs are perturbed by vector and tensor operators, respectively. We find that the same formula holds as in the scalar case. Section 7 is devoted to conclusion and discussion. The Ricci tensor and the scalar curvature are calculated in appendix A.

\section{Information metric in field theory}

We consider a field theory defined on $\mathbb{R}^{d}$, whose coordinates are $(\tau, \vec{x})$, where $\tau$ is the Euclidean time and $\vec{x}$ are the $(d-1)$-dimensional space coordinates. The wave function of the ground state $|\Omega\rangle$ in the theory is represented by a path integral over a time interval from $-\infty$ to 0 as follows:

$$
\langle\tilde{\psi} \mid \Omega\rangle=\frac{1}{Z^{1 / 2}} \int_{\psi(0, \vec{x})=\tilde{\psi}(\vec{x})} \mathcal{D} \psi \exp \left[-\int_{-\infty}^{0} d \tau \int d^{d-1} x \mathcal{L}\right],
$$

where the value of the field $\Psi$ is fixed to $\tilde{\psi}(\vec{x})$ at $\tau=0$, and $Z$ is the partition function of the theory. Note that the ground state is normalized:

$$
\langle\Omega \mid \Omega\rangle=1 .
$$

We further consider two theories, the theory 1 and the theory 2, with the same field content defined by the lagrangians $\mathcal{L}_{1}$ and $\mathcal{L}_{2}$, respectively. We denote the ground states of these two theories by $\left|\Omega_{1}\right\rangle$ and $\left|\Omega_{2}\right\rangle$, respectively. Then, by glueing the wave functions for these ground states given in (2.1), we can represent the inner product between the ground states in terms of a path integral as

$$
\left\langle\Omega_{2} \mid \Omega_{1}\right\rangle=\frac{1}{\left(Z_{1} Z_{2}\right)^{1 / 2}} \int \mathcal{D} \psi \exp \left[-\int d^{d-1} x\left(\int_{-\infty}^{0} d \tau \mathcal{L}_{1}+\int_{0}^{\infty} d \tau \mathcal{L}_{2}\right)\right],
$$

where $Z_{1}$ and $Z_{2}$ are the partition functions of the theory 1 and the theory 2 , respectively. 
We denote the difference of the two lagrangians by $\delta \mathcal{L}$. Namely, we have

$$
\mathcal{L}_{2}=\mathcal{L}_{1}+\delta \mathcal{L}
$$

We rewrite the inner product $(2.3)$ in terms of $\delta \mathcal{L}$ as

$$
\left\langle\Omega_{2} \mid \Omega_{1}\right\rangle=\frac{\left\langle\exp \left[-\int_{0}^{\infty} d \tau \int d^{d-1} x \delta \mathcal{L}\right]\right\rangle_{1}}{\left\langle\exp \left[-\int_{-\infty}^{\infty} d \tau \int d^{d-1} x \delta \mathcal{L}\right]\right\rangle_{1}^{1 / 2}}
$$

where \langle\rangle$_{1}$ stands for the vacuum expectation value taken with respect to the theory 1 :

$$
\langle\mathcal{O}\rangle_{1}=\frac{1}{Z_{1}} \int \mathcal{D} \psi \mathcal{O} e^{-S_{1}}=\left\langle\Omega_{1}|\mathcal{O}| \Omega_{1}\right\rangle
$$

We expand $(2.5)$ in terms of $\delta \mathcal{L}$ up to $\mathcal{O}\left(\delta \mathcal{L}^{2}\right)$ as $[7]$

$$
\left\langle\Omega_{2} \mid \Omega_{1}\right\rangle=1-\frac{1}{2} \int_{0}^{\infty} d \tau \int_{-\infty}^{0} d \tau^{\prime} \int d^{d-1} x \int d^{d-1} x^{\prime}\left\langle\delta \mathcal{L}(\tau, \vec{x}) \delta \mathcal{L}\left(\tau^{\prime}, \vec{x}^{\prime}\right)\right\rangle_{1}
$$

where we have assumed

$$
\langle\delta \mathcal{L}(\tau, \vec{x})\rangle=0
$$

and the time reversal symmetry

$$
\left\langle\delta \mathcal{L}(\tau, \vec{x}) \delta \mathcal{L}\left(\tau^{\prime}, \vec{x}\right)\right\rangle=\left\langle\delta \mathcal{L}(-\tau, \vec{x}) \delta \mathcal{L}\left(-\tau^{\prime}, \vec{x}\right)\right\rangle
$$

We further assume that $\delta \mathcal{L}$ takes the form

$$
\delta \mathcal{L}=\phi(\vec{x}) \mathcal{O}(\tau, \vec{x}),
$$

where $\phi(\vec{x})$ is a source independent of $\tau$ and $\mathcal{O}(\tau, \vec{x})$ is a local operator. Then, using (2.7) and (2.10), we introduce the information metric $\mathcal{G}$ which is known as Fisher's metric and measures the distance between the ground states of the two theories:

$$
\mathcal{G}=\frac{1}{T}\left(1-\left\langle\Omega_{2} \mid \Omega_{1}\right\rangle\right)=\int d^{d-1} x \int d^{d-1} x^{\prime} \mathcal{G}_{\vec{x}, \vec{x}^{\prime}} \phi(\vec{x}) \phi\left(\vec{x}^{\prime}\right)
$$

with

$$
\mathcal{G}_{\vec{x}, \vec{x}^{\prime}}=\frac{1}{2 T} \int_{0}^{\infty} d \tau \int_{-\infty}^{0} d \tau^{\prime}\left\langle\mathcal{O}(\tau, \vec{x}) \mathcal{O}\left(\tau^{\prime}, \vec{x}^{\prime}\right)\right\rangle_{1},
$$

where $T$ is the volume of time direction. 


\section{Information metric as on-shell action}

Let us consider a case in which the theory 1 is a CFT and $\mathcal{O}(\tau, \vec{x})$ in $(2.10)$ is a scalar primary operator with the conformal dimension $\Delta$ in the CFT. Namely, the theory 2 is obtained by perturbing the CFT by the scalar primary operator. In what follows, the quantities in the theory 1 are labeled 'CFT' instead of ' 1 ', while those in the theory 2 have no labels. For instance,

$$
\mathcal{L}=\mathcal{L}_{\mathrm{CFT}}+\phi(\vec{x}) \mathcal{O}(\tau, \vec{x})
$$

and so on.

The one-point function of the primary operator $\mathcal{O}$ vanishes, which implies that $(2.8)$ is satisfied. The two-point function of the primary operator $\mathcal{O}$ takes the form

$$
\left\langle\mathcal{O}(\tau, \vec{x}) \mathcal{O}\left(\tau^{\prime}, \vec{x}^{\prime}\right)\right\rangle_{\mathrm{CFT}}=\frac{C_{\Delta}}{\left(\epsilon^{2}+\left(\tau-\tau^{\prime}\right)^{2}+\left(\vec{x}-\vec{x}^{\prime}\right)^{2}\right)^{\Delta}},
$$

where $C_{\Delta}$ is a normalization constant and a UV cutoff $\epsilon$ has been introduced. We see from (3.2) that (2.9) is satisfied.

The information metric (2.11) and (2.12) reads

$$
\begin{aligned}
\mathcal{G} & =\frac{1}{T}\left(1-\left\langle\Omega \mid \Omega_{\mathrm{CFT}}\right\rangle\right) \\
& =\frac{1}{8} \int_{-\infty}^{\infty} d s \int d^{d-1} x \int d^{d-1} x^{\prime} \frac{C_{\Delta} \phi(\vec{x}) \phi\left(\vec{x}^{\prime}\right)}{\left(\epsilon^{2}+s^{2}+\left(\vec{x}-\vec{x}^{\prime}\right)^{2}\right)^{\Delta}} .
\end{aligned}
$$

Suppose that the CFT has a gravity dual defined on $\mathrm{AdS}_{d+1}$. Throughout this paper, we consider a situation in which the classical approximation is valid on the gravity side. Because the information metric takes the form of the generating functional for the two-point functions, it can be represented by the on-shell action for the bulk field $\Phi$ dual to $\mathcal{O}$.

Here we introduce the following notations: $x^{\mu}=\left(x^{0}, x^{i}\right)=(\tau, \vec{x})$, where $\mu=0, \ldots, d-1$ and $i=1, \ldots, d-1$, and $z^{M}=\left(z, x^{\mu}\right)$. We use the metric of $\mathrm{AdS}_{d+1}$ in the Poincare coordinates, which takes the form

$$
d s^{2}=G_{M N} d z^{M} d z^{N}=\frac{1}{z^{2}}\left(d z^{2}+d x^{\mu} d x^{\mu}\right) .
$$

We define a boundary hypersurface in $\operatorname{AdS}_{d+1}$ by $z=\epsilon$, where $\epsilon$ was introduced in (3.2) as a UV cutoff. The CFT is viewed as defined on the boundary.

The action for $\Phi$ on the gravity side is

$$
S_{M}=\frac{1}{2} \int d^{d+1} x \sqrt{G}\left(G^{M N} \partial_{M} \Phi \partial_{N} \Phi+m^{2} \Phi^{2}\right),
$$

where we have presented only the quadratic terms in $\Phi$, which are needed in the following, and

$$
m^{2}=\Delta(\Delta-d) .
$$


The equation of motion for $\Phi$ is derived from (3.5) as

$$
-\frac{1}{\sqrt{G}} \partial_{M}\left(\sqrt{G} G^{M N} \partial_{N} \Phi\right)+m^{2} \Phi=0 .
$$

The boundary condition for $\Phi$ is given by

$$
\Phi(z=\epsilon, \tau, \vec{x})=\epsilon^{d-\Delta} \phi(\vec{x}) .
$$

The solution to (3.7) satisfying the boundary condition (3.8) [3] is

$$
\Phi(z, x)=\int d^{d} x^{\prime} K\left(z, x-x^{\prime}\right) \phi\left(\vec{x}^{\prime}\right),
$$

where $K$ is the so-called boundary to bulk propagator:

$$
K(z, x)=\frac{\alpha_{\Delta} z^{\Delta}}{\left(z^{2}+x^{2}\right)^{\Delta}} \text { with } \alpha_{\Delta}=\frac{\Gamma(\Delta)}{\pi^{\frac{d}{2}} \Gamma\left(\Delta-\frac{d}{2}\right)} .
$$

Note that $\Phi(z, x)$ is independent of $\tau$.

By substituting (3.9) into (3.5), we evaluate the on-shell action for $\Phi$ as follows:

$$
\begin{aligned}
S_{\text {on-shell }}= & \frac{1}{2} \int d^{d+1} x \partial_{M}\left(\sqrt{G} G^{M N} \Phi \partial_{N} \Phi\right) \\
& -\frac{1}{2} \int d^{d+1} x \sqrt{G} \Phi\left\{\frac{1}{\sqrt{G}} \partial_{M}\left(\sqrt{G} G^{M N} \partial_{N} \Phi\right)-m^{2} \Phi\right\} \\
= & -\frac{1}{2} \int_{z=\epsilon} d^{d} x \epsilon^{-d+1} \Phi \partial_{z} \Phi \\
= & -\Delta \alpha_{\Delta} \int_{0}^{\infty} d \tau \int_{-\infty}^{\infty} d s \int d^{d-1} x d^{d-1} x^{\prime} \frac{\phi(\vec{x}) \phi\left(\vec{x}^{\prime}\right)}{\left(\epsilon^{2}+s^{2}+\left(\vec{x}-\vec{x}^{\prime}\right)^{2}\right)^{\Delta}},
\end{aligned}
$$

where we have used (3.7) to obtain the third equality. This would be the generating functional of $\phi(\vec{x})$ for two-point functions of $\mathcal{O}$ so that we obtain

$$
C_{\Delta}=\Delta \alpha_{\Delta}
$$

Then, by comparing (3.11) with (3.3), we find

$$
S_{\text {on-shell }}=-4 T \mathcal{G}
$$

\section{Back reaction to the AdS geometry}

The theory (3.1) obtained by perturbing the CFT by the primary operator would have a gravity dual where the geometry has a back reaction to the AdS geometry, namely deviates from the AdS geometry. We evaluate the back reaction up to $\mathcal{O}\left(\phi^{2}\right)$ in the following.

We parametrize the metric with the back reaction as

$$
d s^{2}=G_{M N} d z^{M} d z^{N}=\frac{1}{z^{2}}\left(d z^{2}+g_{\mu \nu}(z, x) d x^{\mu} d x^{\nu}\right),
$$


with

$$
g_{\mu \nu}(z, x)=\delta_{\mu \nu}+h_{\mu \nu}(z, x),
$$

where $h_{\mu \nu}$ represent the back reaction to the AdS geometry and start with $\mathcal{O}\left(\phi^{2}\right)$ contribution, which we will focus on.

The gravity action on the gravity side is given by

$$
S_{G}=\frac{1}{16 \pi G_{N}}\left[\int d^{d+1} x \sqrt{G}(-R[G]+2 \Lambda)-\int_{z=\epsilon} d^{d} x \sqrt{\gamma}(2 K+\lambda)\right],
$$

where the cosmological constant $\Lambda$ is $\Lambda=\frac{-d(d-1)}{2}$, the boundary cosmological constant $\lambda$ is $\lambda=-2(d-1), \gamma$ is the induced metric on the boundary, and $K$ is the trace of the extrinsic curvature. The scalar curvature $R[G]$ is defined in (A.8).

The Einstein equation is derived from (4.3) and (3.5) as

$$
R[G]_{M N}+d G_{M N}=8 \pi G_{N} \mathcal{T}_{M N}
$$

where the Ricci tensor $R[G]_{M N}$ are defined in (A.7), and $\mathcal{T}_{M N}$ are defined as

$$
\mathcal{T}_{M N} \equiv T_{M N}-\frac{1}{d-1} G_{M N} G^{I J} T_{I J}
$$

with

$$
T_{M N}=\partial_{M} \Phi \partial_{N} \Phi-\frac{1}{2} G_{M N}\left(\partial_{L} \Phi \partial^{L} \Phi+m^{2} \Phi^{2}\right)
$$

Each component of (4.4) takes the following form:

$$
\begin{aligned}
& \operatorname{Tr} g^{-1} g^{\prime \prime}-\frac{1}{z} \operatorname{Tr} g^{-1} g^{\prime}-\frac{1}{2} \operatorname{Tr} g^{-1} g^{\prime} g^{-1} g^{\prime}=-16 \pi G_{N} \mathcal{T}_{z z} \\
& \nabla_{\mu} \operatorname{Tr} g^{-1} g^{\prime}-\nabla^{\lambda} g_{\lambda \mu}^{\prime}=-16 \pi G_{N} \mathcal{T}_{z \mu} \\
& g_{\mu \nu}^{\prime \prime}-g_{\mu \lambda}^{\prime} g^{\lambda \sigma} g_{\sigma \nu}^{\prime}+\frac{1}{2} \operatorname{Tr}\left(g^{-1} g^{\prime}\right) g_{\mu \nu}^{\prime}-(d-1) \frac{1}{z} g_{\mu \nu}^{\prime}-\frac{1}{z} \operatorname{Tr}\left(g^{-1} g^{\prime}\right) g_{\mu \nu}-2 \operatorname{Ric}_{\mu \nu}(g) \\
& \quad=-16 \pi G_{N} \mathcal{T}_{\mu \nu}
\end{aligned}
$$

with

$$
\begin{aligned}
& \mathcal{T}_{z z}=\partial_{z} \Phi \partial_{z} \Phi+\frac{m^{2}}{d-1} \frac{1}{z^{2}} \Phi^{2}, \\
& \mathcal{T}_{z \mu}=\partial_{z} \Phi \partial_{\mu} \Phi, \\
& \mathcal{T}_{\mu \nu}=\partial_{\mu} \Phi \partial_{\nu} \Phi+\frac{m^{2}}{d-1} \frac{1}{z^{2}} g_{\mu \nu} \Phi^{2},
\end{aligned}
$$

where the prime stands for the derivative with respect to $z, \operatorname{Tr}$ is defined by $\operatorname{Tr} A=\delta^{\mu \nu} A_{\mu \nu}$, $\nabla_{\mu}$ is the covariant derivative with respect to the metric $g_{\mu \nu}$, and $\operatorname{Ric}_{\mu \nu}[g]$ are the Ricci tensor for $g_{\mu \nu}$. 
We expand the left hand sides up to $\mathcal{O}\left(h_{\mu \nu}\right)$ in order to evaluate $h_{\mu \nu}$ up to $\mathcal{O}\left(\phi^{2}\right)$, since the righthand sides of (4.7), (4.8) and (4.9) are $\mathcal{O}\left(\phi^{2}\right)$. Here, $h_{\mu \nu}$ is independent of $\tau$, because so is $\Phi$, and we ignore total derivative terms with respect to $\vec{x}$, which will be justified shortly. Then, (4.7) reduces to

$$
\operatorname{Tr} h^{\prime \prime}-\frac{1}{z} \operatorname{Tr} h^{\prime}=-16 \pi G_{N}\left(\partial_{z} \Phi \partial_{z} \Phi+\frac{m^{2}}{d-1} \frac{1}{z^{2}} \Phi^{2}\right),
$$

while (4.9) reduces to

$$
h_{\mu \nu}^{\prime \prime}-(d-1) \frac{1}{z} h_{\mu \nu}^{\prime}-\frac{1}{z} \operatorname{Tr}^{\prime} \delta_{\mu \nu}=-16 \pi G_{N}\left(\partial_{\mu} \Phi \partial_{\nu} \Phi+\frac{m^{2}}{d-1} \frac{1}{z^{2}} \delta_{\mu \nu} \Phi^{2}\right) .
$$

Taking the trace of (4.12) yields

$$
\operatorname{Tr} h^{\prime \prime}-(2 d-1) \frac{1}{z} \operatorname{Tr} h^{\prime}=-16 \pi G_{N}\left(\partial_{\mu} \Phi \partial_{\mu} \Phi+\frac{m^{2}}{d-1} \frac{d}{z^{2}} \Phi^{2}\right) .
$$

The 00 component of (4.12) reads

$$
h_{00}^{\prime \prime}-(d-1) \frac{1}{z} h_{00}^{\prime}-\frac{1}{z} \operatorname{Tr} h^{\prime}=-16 \pi G_{N} \frac{m^{2}}{d-1} \frac{1}{z^{2}} \Phi^{2} .
$$

By taking a linear combination of (4.11), (4.13) and (4.14), we obtain

$$
\operatorname{tr} h^{\prime \prime}-\frac{d-1}{z} \operatorname{tr} h^{\prime}=-8 \pi G_{N}\left\{\partial_{z}\left(\Phi \partial_{z} \Phi\right)-\frac{d-1}{z} \Phi \partial_{z} \Phi\right\},
$$

where $\operatorname{tr} A=A_{i i}=\operatorname{Tr} A-A_{00}$, and we have used (3.7) and again ignored the total derivative terms with respect to $\vec{x}$. Integrating (4.15) leads to

$$
\operatorname{tr} h^{\prime}=-8 \pi G_{N} \Phi \partial_{z} \Phi,
$$

where the boundary condition $\lim _{z \rightarrow \infty} h_{\mu \nu}=0$ has been used.

By using the third equality in (3.11) and (4.16), we obtain

$$
S_{\text {on-shell }}=\frac{1}{16 \pi G_{N}} \int_{z=\epsilon} d^{d} x \epsilon^{-d+1} \operatorname{tr} h^{\prime} .
$$

Here ignoring the total derivative terms in deriving (4.16) is justified.

By comparing (3.13) and (4.17), we find a formula

$$
\mathcal{G}=-\frac{1}{64 \pi G_{N}} \int_{z=\epsilon} d^{d-1} x \epsilon^{-d+1} \operatorname{tr} h^{\prime} .
$$

This formula represents the information metric in field theory in terms of buck reaction to the AdS bulk geometry. The righthand side of (4.18) is interpreted geometrically as follows.

We consider a codimension-2 hypersurface specified by $z=\epsilon$ and $\tau=$ const. (see figure 1). The induced metric on the hypersurface in the static gauge is given by

$$
\begin{aligned}
\gamma_{i j} & =\frac{\partial z^{M}}{\partial x^{i}} \frac{\partial z^{N}}{\partial x^{j}} G_{M N} \\
& =\frac{1}{z^{2}} g_{i j} .
\end{aligned}
$$




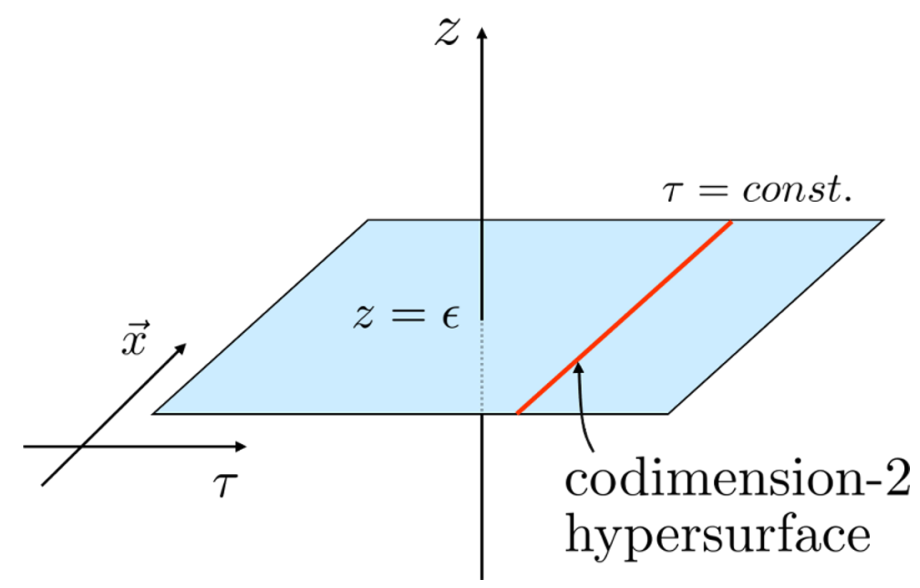

Figure 1. A red line represents a codimension-2 hypersurface.

The volume of the hypersurface is evaluated up to $\mathcal{O}\left(h_{i j}\right)$ as

$$
\begin{aligned}
v & =\int_{z=\epsilon} d^{d-1} x \sqrt{\gamma} \\
& =\int_{z=\epsilon} d^{d-1} x z^{-d+1} \sqrt{\operatorname{det} g_{i j}} \\
& =\int_{z=\epsilon} d^{d-1} x z^{-d+1}\left(1+\frac{1}{2} \operatorname{tr} h\right) .
\end{aligned}
$$

We subtract the volume of the hypersurface in the AdS case where $h_{i j}=0$ and denote the difference by $\delta v$ :

$$
\delta v=\frac{1}{2} \int_{z=\epsilon} d^{d-1} x z^{-d+1} \operatorname{trh} .
$$

By taking the derivative with respect to $z$, we obtain

$$
\delta v^{\prime}=\frac{1}{2} \int_{z=\epsilon} d^{d-1} x\left((-d+1) z^{-d} \operatorname{tr} h+z^{-d+1} \operatorname{tr} h^{\prime}\right) .
$$

While the first term in (4.22) represents the canonical scaling of the volume, the second term represents a nontrivial scaling of the volume and is proportional to the righthand side of (4.18). Thus, (4.18) is rewritten as

$$
\mathcal{G}=-\frac{1}{32 \pi G_{N}} \delta v_{\text {nontrivial }}^{\prime}
$$

with

$$
\delta v_{\text {nontrivial }}^{\prime}=\frac{1}{2} \int_{z=\epsilon} d^{d-1} x z^{-d+1} \operatorname{tr} h^{\prime}
$$




\section{$5 \quad$ Vector field}

In this section, we extend the analysis in sections 3 and 4 to the case of vector field.

We perturb a CFT by a U(1) vector current $\mathcal{J}^{\mu}(x)$ : the counterpart of the Lagrangian (3.1) is given by

$$
\mathcal{L}=\mathcal{L}_{\mathrm{CFT}}+a_{\mu}(\vec{x}) \mathcal{J}^{\mu}(x),
$$

where the source $a_{\mu}(\vec{x})$ is independent of the time, and $a_{0}=0$. The 2-point function of $\mathcal{J}^{\mu}(x)$ is given by

$$
\left\langle\mathcal{J}_{\mu}(\tau, \vec{x}) \mathcal{J}_{\nu}\left(\tau^{\prime}, \vec{x}^{\prime}\right)\right\rangle=\frac{C_{V}}{\left(\epsilon^{2}+\left|x-x^{\prime}\right|^{2}\right)^{d-1}} J_{\mu \nu}\left(\epsilon, \tau-\tau^{\prime}, \vec{x}-\vec{x}^{\prime}\right),
$$

where

$$
J_{\mu \nu}\left(\epsilon, \tau-\tau^{\prime}, \vec{x}-\vec{x}^{\prime}\right)=\delta_{\mu \nu}-\frac{2\left(x-x^{\prime}\right)_{\mu}\left(x-x^{\prime}\right)_{\nu}}{\epsilon^{2}+\left|x-x^{\prime}\right|^{2}},
$$

and $C_{V}$ is a normalization constant. Note that (2.8) and (2.9) are satisfied.

The information metric, which is the counterpart of (3.3), is

$$
\mathcal{G}=\frac{C_{V}}{8} \int_{\infty}^{\infty} d s \int d^{d-1} x \int d^{d-1} x^{\prime} a^{i}(\vec{x}) a^{j}\left(\vec{x}^{\prime}\right) \frac{J_{i j}\left(\epsilon, s, \vec{x}-\vec{x}^{\prime}\right)}{\left(\epsilon^{2}+s^{2}+\left(\vec{x}-\vec{x}^{\prime}\right)^{2}\right)^{d-1}} .
$$

A bulk field corresponding to $\mathcal{J}_{\mu}$ is a $\mathrm{U}(1)$ gauge field $A_{M}$. The bulk action $S_{A}$ for $A_{M}$ is given by

$$
S_{A}=\frac{1}{4} \int d^{d+1} x \sqrt{G} F^{M N} F_{M N},
$$

where $F_{M N}=\partial_{M} A_{N}-\partial_{N} A_{M}$. The equations of motion for $A_{M}$ derived from (5.5) are

$$
\frac{1}{\sqrt{G}} \partial_{M}\left(\sqrt{G} F^{M N}\right)=0 .
$$

Solving these equations around the AdS metric (3.4) with a gauge $A_{z}=0$ and a boundary condition $A_{\mu}(\epsilon, x)=a_{\mu}(\vec{x})$ leads to [3]

$$
A_{\mu}(z, x)=\alpha_{V} \int d^{d} x^{\prime} \frac{z^{d-2}}{\left(z^{2}+\left|x-x^{\prime}\right|^{2}\right)^{d-1}} J_{\mu}^{i}\left(z, \tau-\tau^{\prime}, \vec{x}-\vec{x}^{\prime}\right) a_{i}\left(\vec{x}^{\prime}\right)
$$

with

$$
\alpha_{V}=\frac{1}{2} \frac{\Gamma(d)}{\pi^{\frac{d}{2}} \Gamma\left(\frac{d}{2}\right)} .
$$

Note that $A_{\mu}(z, x)$ is independent of $\tau$ and that $A_{0}=0$.

By substituting (5.7) into (5.5), the on-shell action is obtained as

$$
\begin{aligned}
S_{A, \text { on-shell }} & =\frac{1}{2} \int d^{d+1} x \partial_{M}\left(\sqrt{G} G^{K M} G^{L N} A_{N} F_{K L}\right)-\frac{1}{2} \int d^{d+1} x A_{N} \partial_{M}\left(\sqrt{G} F^{M N}\right) \\
& =-\frac{1}{2} \int_{z=\epsilon} d^{d} x z^{-d+3} A^{i} F_{z i} \\
& =-\frac{(d-2) \alpha_{V}}{2} \int_{-\infty}^{\infty} d s \int d^{d-1} x d^{d-1} x^{\prime} a_{i}(\vec{x}) \frac{J_{i j}\left(\epsilon, s, \vec{x}-\vec{x}^{\prime}\right)}{\left(\epsilon^{2}+s^{2}+\left|\vec{x}-\vec{x}^{\prime}\right|^{2}\right)^{d-1}} a_{j}\left(\vec{x}^{\prime}\right) .
\end{aligned}
$$


This on-shell action is the generating functional for the 2-point function for (5.2) so that $C_{V}$ is determined as $C_{V}=(d-2) \alpha_{V}$. By comparing (5.4) and (5.9), we again obtain (3.13).

We consider the back reaction to the AdS geometry. The bulk action consists of the gravity part (4.3) and the gauge field part (5.5). The Einstein equations derived from the bulk action are (4.7), (4.8) and (4.9) with

$$
\begin{aligned}
& \mathcal{T}_{z z}=\frac{d-2}{d-1} z^{2} g^{\mu \nu} F_{z \mu} F_{z \nu}-\frac{1}{2(d-1)} z^{2} F_{\mu \nu} F_{\mu \nu}, \\
& \mathcal{T}_{z \mu}=z^{2} g^{\alpha \beta} F_{z \alpha} F_{\mu \beta}, \\
& \mathcal{T}_{\mu \nu}=z^{2}\left(F_{z \mu} F_{z \nu}-\frac{1}{d-1} g_{\mu \nu} g^{\alpha \beta} F_{z \alpha} F_{z \beta}+g^{\alpha \beta} F_{\mu \alpha} F_{\nu \beta}-\frac{1}{2(d-1)} g_{\mu \nu} F^{\alpha \beta} F_{\alpha \beta}\right) .
\end{aligned}
$$

We again expand the metric around the AdS metric as (4.2). Then, the counterpart of $(4.11)$ is

$$
\operatorname{Tr} h^{\prime \prime}-\frac{1}{z} \operatorname{Tr} h^{\prime}=-16 \pi G_{N}\left(\frac{d-2}{d-1} z^{2} \delta^{\mu \nu} F_{z \mu} F_{z \nu}-\frac{1}{2(d-1)} z^{2} F_{\mu \nu} F_{\mu \nu}\right),
$$

while the counterpart of (4.12) is

$$
\begin{aligned}
& h_{\mu \nu}^{\prime \prime}-(d-1) \frac{1}{z} h_{\mu \nu}^{\prime}-\frac{1}{z} \operatorname{Tr} h^{\prime} \delta_{\mu \nu} \\
& =-16 \pi G_{N}\left(F_{z \mu} F_{z \nu}-\frac{1}{d-1} \delta_{\mu \nu} \delta^{\alpha \beta} F_{z \alpha} F_{z \beta}+\delta^{\alpha \beta} F_{\mu \alpha} F_{\nu \beta}-\frac{1}{2(d-1)} \delta_{\mu \nu} F^{\alpha \beta} F_{\alpha \beta}\right) .
\end{aligned}
$$

The trace part of $(5.12)$ is

$$
\operatorname{Tr} h^{\prime \prime}-(2 d-1) \frac{1}{z} \operatorname{Tr} h^{\prime}=-16 \pi G_{N} z^{2}\left(\frac{1}{1-d} \delta^{\mu \nu} F_{z \mu} F_{z \nu}+\frac{d-2}{2(d-1)} F^{\mu \nu} F_{\mu \nu}\right),
$$

and the 00 component of $(5.12)$ is

$$
\begin{aligned}
& h_{00}^{\prime \prime}-(d-1) \frac{1}{z} h_{00}^{\prime}-\frac{1}{z} \operatorname{Tr} h^{\prime} \\
& =-16 \pi G_{N}\left(F_{z 0} F_{z 0}-\frac{1}{d-1} \delta^{\alpha \beta} F_{z \alpha} F_{z \beta}+\delta^{\alpha \beta} F_{0 \alpha} F_{0 \beta}-\frac{1}{2(d-1)} F^{\alpha \beta} F_{\alpha \beta}\right) .
\end{aligned}
$$

Taking an appropriate linear combination of (5.11), (5.13) and (5.14) and using the equations of motion, we obtain

$$
\begin{aligned}
& \operatorname{tr} h^{\prime \prime}-\frac{d-1}{z} \operatorname{tr} h^{\prime} \\
& =-16 \pi G_{N}\left\{\frac{1}{2} \partial_{z}\left(z^{2} A^{\alpha} F_{z \alpha}\right)-\frac{d-1}{2} z A^{\alpha} F_{z \alpha}-\partial_{z}\left(z^{2} A_{0} \partial_{z} A_{0}\right)+(d-1) z A_{0} \partial_{z} A_{0}\right\},
\end{aligned}
$$

where we have ignored the total derivative terms with respect to $\vec{x}$. Integrating (5.15) leads to

$$
\operatorname{tr} h^{\prime}=-8 \pi G_{N} z^{2} A^{i} F_{z i}
$$

where the boundary condition $\lim _{z \rightarrow \infty} h_{\mu \nu}=0$ has been used again.

From (5.9) and (5.16), we again obtain (4.17). Thus, since we have (3.13) and (4.17), we obtain the same formula (4.23) as in the case of scalar field. 


\section{Tensor field}

Finally, let us consider the case in which a CFT is perturbed by the energy momentum tensor:

$$
\mathcal{L}=\mathcal{L}_{\mathrm{CFT}}+\hat{h}_{\mu \nu}(\vec{x}) T^{\mu \nu}(x),
$$

where the source $\hat{h}_{\mu \nu}$ is independent of the time and $\hat{h}_{0 \mu}=0$. Since $T_{\mu \mu}=0$, we can assume without loss of generality that $\hat{h}_{\mu \mu}=0$. The 2-point function of $T_{\mu \nu}$ is given by

$$
\left\langle T_{\mu \nu}(\tau, \vec{x}) T_{\rho \sigma}\left(\tau^{\prime}, \vec{x}^{\prime}\right)\right\rangle=\frac{C_{T} P_{\alpha \beta \rho \sigma}}{\left(\epsilon^{2}+\left|x-x^{\prime}\right|^{2}\right)^{d-1}} J_{\mu \alpha}\left(\epsilon, \tau-\tau^{\prime}, \vec{x}-\vec{x}^{\prime}\right) J_{\nu \beta}\left(\epsilon, \tau-\tau^{\prime}, \vec{x}-\vec{x}^{\prime}\right),
$$

where

$$
\begin{aligned}
J_{\mu \nu}\left(\epsilon, \tau-\tau^{\prime}, \vec{x}-\vec{x}^{\prime}\right) & =\delta_{\mu \nu}-\frac{2\left(x-x^{\prime}\right)_{\mu}\left(x-x^{\prime}\right)_{\nu}}{\epsilon^{2}+\left|x-x^{\prime}\right|^{2}}, \\
P_{\alpha \beta \rho \sigma} & =\frac{1}{2}\left(\delta_{\alpha \rho} \delta_{\beta \sigma}+\delta_{\alpha \sigma} \delta_{\beta \rho}\right)-\frac{1}{d} \delta_{\alpha \beta} \delta_{\rho \sigma},
\end{aligned}
$$

and $C_{T}$ is a normalization constant. Note that (2.8) and (2.9) are satisfied.

The information metric, which is the counterpart of (3.3), is

$$
\begin{aligned}
\mathcal{G} & =\frac{1}{2 T} \int_{0}^{\infty} d \tau \int_{-\infty}^{0} d \tau^{\prime} \int d^{d-1} x \int d^{d-1} x^{\prime} \hat{h}^{i j}(\vec{x})\left\langle T_{i j}(\tau, \vec{x}) T_{k l}\left(\tau^{\prime}, \vec{x}^{\prime}\right)\right\rangle \hat{h}^{k l}\left(\vec{x}^{\prime}\right) \\
& =\frac{C_{T}}{8} \int_{-\infty}^{\infty} d s \int d^{d-1} x \int d^{d-1} x^{\prime} \hat{h}^{i j}(\vec{x}) \hat{h}^{k l}\left(\vec{x}^{\prime}\right) \frac{J_{i \alpha}\left(\epsilon, s, \vec{x}-\vec{x}^{\prime}\right) J_{j \beta}\left(\epsilon, s, \vec{x}-\vec{x}^{\prime}\right) P_{\alpha \beta k l}}{\left(\epsilon^{2}+s^{2}+\left(\vec{x}-\vec{x}^{\prime}\right)^{2}\right)^{d-1}} .
\end{aligned}
$$

The bulk field corresponding to $T_{\mu \nu}$ is $h_{\mu \nu}$ in (4.2). We solve the Einstein equation derived from $S_{G}(4.3)$ with respect to $h_{\mu \nu}$. The boundary condition for $h_{\mu \nu}(\epsilon, x)=\hat{h}_{\mu \nu}(\vec{x})$. We expand $h_{\mu \nu}$ as

$$
h_{\mu \nu}=h_{(1) \mu \nu}+h_{(2) \mu \nu}+\cdots,
$$

where $h_{(1) \mu \nu}$ and $h_{(2) \mu \nu}$ are contributions of the first and second orders in $\hat{h}_{\mu \nu}$, respectively. Putting $\mathcal{T}_{M N}=0$ in (4.7), (4.8) and (4.9). yields the Einstein equation in this case.

By expanding the Einstein equation up to $\mathcal{O}\left(h_{(1) \mu \nu}\right)$, we obtain the equations for $h_{(1) \mu \nu}$ : (4.7) reduces to

$$
\operatorname{Tr} h_{(1)}^{\prime \prime}-\frac{1}{z} \operatorname{Tr} h_{(1)}^{\prime}=0 .
$$

(4.8) reduces to

$$
\left(\partial_{\mu} \operatorname{Tr} h_{(1)}-\partial_{\nu} h_{(1)}^{\nu}\right)^{\prime}=0
$$

(4.9) reduces to

$$
h_{(1) \mu \nu}^{\prime \prime}-(d-1) \frac{1}{z} h_{(1) \mu \nu}^{\prime}-\frac{1}{z} \operatorname{Tr} h_{(1)}^{\prime} \delta_{\mu \nu}=\partial^{\alpha}\left(\partial_{\mu} h_{(1) \nu \alpha}+\partial_{\nu} h_{(1) \mu \alpha}-\partial_{\alpha} h_{(1) \mu \nu}\right)-\partial_{\mu} \partial_{\nu} \operatorname{Tr} h_{(1)} .
$$


The solution to this equation is given by

$$
\operatorname{Tr} h_{(1)}^{\prime}=0, \quad \operatorname{Tr} h_{(1)}=0 .
$$

The solution to (6.9), (4.8) and (4.9) with the boundary condition $h_{(1) \mu \nu}(\epsilon, x)=\hat{h}_{\mu \nu}(\vec{x})$ is given by $[16]$

$$
\begin{aligned}
h_{(1) \mu \nu}(z, x)=\alpha_{T} \int & d^{d} x^{\prime} \frac{z^{d}}{\left(z^{2}+\left|x-x^{\prime}\right|^{2}\right)^{d}} J_{\mu \rho}\left(z, \tau-\tau^{\prime}, \vec{x}-\vec{x}^{\prime}\right) J_{\nu \sigma}\left(z, \tau-\tau^{\prime}, \vec{x}-\vec{x}^{\prime}\right) \\
& \times P_{\rho \sigma i j} \hat{h}_{i j}\left(\vec{x}^{\prime}\right),
\end{aligned}
$$

with

$$
\alpha_{T}=\frac{d+1}{d-1} \frac{\Gamma(d)}{\pi^{\frac{d}{2}} \Gamma\left(\frac{d}{2}\right)} .
$$

Note that $h_{\mu \nu}(z, x)$ is independent of $\tau$ and that $h_{0 i}=0$.

By using (6.6) and (6.8), we obtain the on-shell action for the gravitational field $h_{(1) \mu \nu}$ :

$$
S_{G, \text { on-shell }}=\frac{1}{16 \pi G_{N}} \int_{z=\epsilon} d^{d} x z^{-d+1}\left\{-\frac{1}{4} h_{(1)}{ }^{i j} h_{(1)_{i j}^{\prime}}^{\prime}-\frac{1}{4} h_{(1)} h_{00}{ }_{(1)_{00}^{\prime}}\right\}
$$

Substituting (6.10) into (6.12) yields

$$
S_{G, \text { on-shell }}=-\frac{d \alpha_{T} T}{64 \pi G_{N}} \int_{-\infty}^{\infty} d s \int d^{d-1} x d^{d-1} x^{\prime} \hat{h}_{i j}(\vec{x}) \frac{J_{i \alpha}\left(\epsilon, s, \vec{x}-\vec{x}^{\prime}\right) J_{j \beta}\left(\epsilon, s, \vec{x}-\vec{x}^{\prime}\right) P_{\alpha \beta k l}}{\left(\epsilon^{2}+s^{2}+\left|\vec{x}-\vec{x}^{\prime}\right|^{2}\right)^{d-1}}\left(\vec{x}^{\prime}\right),
$$

which implies that $C_{T}=\frac{d \alpha_{T}}{32 \pi G_{N}}$. We find the same relation (3.13) between the information metric and the on-shell action.

Next, we consider the equations for the back reaction $h_{(2)}$. In what follows, we ignore total derivatives with respect to $\vec{x}$. (4.7) reduces to

$$
\operatorname{Tr} h_{(2)}^{\prime \prime}-\frac{1}{z} \operatorname{Tr} h_{(2)}^{\prime}=h_{(1)}^{\mu \nu} h_{(1)}^{\prime \prime}{ }_{\mu \nu}-\frac{1}{z} h_{(1)}{ }^{\mu \nu} h_{(1)}^{\prime}{ }_{\mu \nu}^{\prime}+\frac{1}{2} h_{(1)}^{\prime}{ }^{\mu \nu} h_{(1)}^{\prime}{ }_{\mu \nu}^{\prime}
$$

while (4.9) reduces to

$$
\begin{aligned}
& h_{(2) \mu \nu}^{\prime \prime}-(d-1) \frac{1}{z} h_{(2) \mu \nu}^{\prime}-\frac{1}{z} \operatorname{Tr} h_{(2)}^{\prime} \delta_{\mu \nu}-2 \operatorname{Ric}^{(1)}\left(h_{(2)}\right)_{\mu \nu} \\
& =h_{(1)_{\mu \alpha}^{\prime}} \delta^{\alpha \beta} h_{(1)_{\beta \nu}^{\prime}}^{\prime}-\frac{1}{z} h_{(1)}^{\alpha \beta} h_{(1)_{\alpha \beta}^{\prime}}^{\prime} \delta_{\mu \nu}-\frac{1}{2} \operatorname{Tr}_{(1)}^{\prime} h_{\mu \nu}^{\prime}+\frac{1}{z} \operatorname{Tr}^{\prime} h_{\mu \nu}+2 \operatorname{Ric}^{(2)}\left(h_{(1)}\right)_{\mu \nu}
\end{aligned}
$$

The trace part and the 00 component of (6.15) takes the forms

$$
\begin{aligned}
& \operatorname{Tr} h_{(2)}^{\prime \prime}-(2 d-1) \frac{1}{z} \operatorname{Tr} h_{(2)}^{\prime}=\frac{1}{2} h_{(1)}{ }^{\mu \nu} h_{(1)}{ }_{\mu \nu}^{\prime \prime}+h_{(1)}{ }^{\prime \mu \nu} h_{(1)_{\mu \nu}^{\prime}}^{\prime}-\frac{3 d-1}{2 z} h_{(1)}{ }^{\mu \nu} h_{(1)}^{\prime}{ }_{\mu \nu}^{\prime} \text {, } \\
& h_{(2) 00}^{\prime \prime}-(d-1) \frac{1}{z} h_{(2) 00}^{\prime}-\frac{1}{z} \operatorname{Tr} h_{(2)}^{\prime}=h_{(1)_{0}^{\alpha}}^{\alpha} h_{(1)_{0 \alpha}}^{\prime \prime}+h_{(1)_{0}}^{\prime} h_{(1)_{0 \alpha}}^{\prime}-\frac{d-1}{z} h_{(1)_{0}^{\alpha}}^{\alpha} h_{(1)_{0 \alpha}}^{\prime} \\
& -\frac{1}{z} h_{(1)}^{\mu \nu} h_{(1)}{ }_{\mu \nu}
\end{aligned}
$$


respectively, and (6.6), (6.7), (6.8) and (6.9) have been used. Taking appropriate linear combinations of $(6.14),(6.16)$ and $(6.17)$, we obtain an equation for $\operatorname{tr} h_{(2)}^{\prime}=\delta^{i j} h_{(2) i j}^{\prime}$ :

$$
\operatorname{tr} h_{(2)}^{\prime}=\frac{3}{4} h_{(1)}{ }^{i j} h_{(1)_{i j}}^{\prime}-\frac{1}{4} h_{(1)} h_{00}^{\prime}{ }_{(1)_{00}} .
$$

By using (6.18), the gravity on-shell action (6.12) is rewritten as

$$
S_{G, \text { on-shell }}=\frac{1}{16 \pi G_{N}} \int_{z=\epsilon} d^{d} x z^{-d+1}\left\{\operatorname{tr} h_{(2)}^{\prime}-h_{(1)}^{i j} h_{(1)_{i j}^{\prime}}^{\prime}\right\} .
$$

The first term comes from the $S_{\mathrm{EH}}$, being a counterpart of (4.17).

The induced metric of a hypersurface with $\tau$ and $z$ fixed is given by $\gamma_{i j}=z^{-2}\left(\delta_{i j}+\right.$ $\left.h_{(1) i j}+h_{(2) i j}\right)$ The volume of the hypersurface is

$$
\begin{aligned}
v & =\int d^{d-1} x \sqrt{\gamma} \\
& =\int d^{d-1} x z^{-d+1}\left(1-\frac{1}{4} h_{(1)}^{i j} h_{(1)_{i j}}+\frac{1}{2} \operatorname{tr} h_{(2)}\right)
\end{aligned}
$$

The counterpart of (4.22) is given by

$$
\delta v^{\prime}=\frac{1}{2} \int d^{d-1} x\left\{(-d+1) z^{-d}\left(\operatorname{trh}_{(2)}-\frac{1}{2} h_{(1)}{ }^{i j} h_{(1)_{i j}}\right)+z^{-d+1}\left(\operatorname{tr}_{(2)}^{\prime}-h_{(1)}{ }^{i j} h_{(1)}{ }_{i j}^{\prime}\right)\right\}
$$

While the first and second terms in (6.21) represent the canonical scaling of the volume, the third and fourth terms represent a nontrivial scaling of the volume and is proportional to the righthand side of (6.19). Thus, from (6.19), we again obtain (4.23) with (4.24) replaced by

$$
\delta v_{\text {nontrivial }}^{\prime}=\frac{1}{2} \int_{z=\epsilon} d^{d-1} x z^{-d+1}\left(\operatorname{tr}_{(2)}^{\prime}-h_{(1)}{ }^{i j} h_{(1)}^{\prime}{ }_{i j}^{\prime}\right) .
$$

\section{Conclusion and discussion}

In this paper, we studied how information geometry is described by bulk geometry. We considered a quantum information metric that measures the distance between the ground states of a CFT and a theory obtained by perturbating the CFT. We represented the information metric in terms of the back reaction that the bulk geometry gains due to the perturbation. We found the formula (4.23) that expresses the information metric by deviation of the volume of the hypersurface in the bulk from that in the AdS case. The geometrical quantity is local in the bulk direction. This formula is universal in the sense that it holds for all the cases of scalar, vector and tensor perturbations. It associates information geometry with dynamics of gravity. The information metric is related to a codimension-2 hypersurface in this paper, while it is related to a codimension-1 hypersurface in [7-11]. This difference comes from one between the situations considered on the gravity side as mentioned in section 1 . 
We associated the information metric with the volume of a hypersurface specified by $z=\epsilon$ with $\epsilon$ small and $\tau=$ const. . In order to reconstruct full bulk geometry from field theory, we should associate it with a hypersurface specified by $z=$ an arbitrary constant by using renormalization group. Furthermore, it is needed to find relationship between information geometry and bulk quantities local even in $\vec{x}$ directions. To understand the geometrical meaning of the formula (4.23) more deeply, we should find how general codimension-2 hypersurfaces in bulk are related to information geometry. It is also relevant to derive effects of strings and quantum gravity from information geometry to construct quantum theory of gravity. We hope to report progress in these issues in the near future.

\section{Acknowledgments}

A.T. was supported in part by Grant-in-Aid for Scientific Research (No. 18K03614) from Japan Society for the Promotion of Science.

\section{A Ricci tensor and scalar curvature}

In this appendix, we calculate the Ricci tensor and the scalar curvature for the metric (4.1).

The Christoffel symbols are given by

$$
\begin{aligned}
\Gamma_{z z}^{z} & =-\frac{1}{z^{2}}, \\
\Gamma_{\alpha \beta}^{z} & =\frac{1}{z} g_{\alpha \beta}-\frac{1}{2} g_{\alpha \beta}^{\prime}, \\
\Gamma_{z \gamma}^{\alpha} & =\frac{1}{2} g^{\alpha \beta} g_{\beta \gamma}^{\prime}-\frac{1}{z} \delta_{\gamma}^{\alpha}, \\
\Gamma_{\beta \gamma}^{\alpha} & =\frac{1}{2} g^{\alpha \delta}\left(\partial_{\beta} g_{\gamma \delta}+\partial_{\gamma} g_{\beta \delta}-\partial_{\delta} g_{\beta \gamma}\right) \\
& =\Gamma_{\beta \gamma}^{\alpha}(g), \\
\Gamma_{\alpha z}^{z} & =\Gamma_{z z}^{\alpha}=0 .
\end{aligned}
$$

The Riemann curvature, the Ricci tensor and the scalar curvature are defined by

$$
\begin{aligned}
R_{J K L}^{I}[G] & =\partial_{K} \Gamma_{J L}^{I}-\partial_{L} \Gamma_{J K}^{I}+\Gamma_{M K}^{I} \Gamma_{L J}^{M}-\Gamma_{M L}^{I} \Gamma_{K J}^{M} \\
R_{M N}[G] & =R_{M K N}^{K}[G] \\
R[G] & =G^{M N} R_{M N}[G]
\end{aligned}
$$

respectively.

The components of the Ricci tensor are given by

$$
\begin{aligned}
R[G]_{z z} & =-\frac{1}{2}\left(\operatorname{Tr} g^{-1} g^{\prime \prime}-\frac{1}{z} \operatorname{Tr} g^{-1} g^{\prime}-\frac{1}{2} \operatorname{Tr} g^{-1} g^{\prime} g^{-1} g^{\prime}+\frac{2}{z^{2}}\right), \\
R[G]_{z \mu} & =\frac{1}{2}\left(\nabla^{\alpha} g_{\alpha \mu}^{\prime}-\nabla_{\mu} \operatorname{Tr} g^{-1} g^{\prime}\right), \\
R[G]_{\mu \nu}= & -\frac{1}{2}\left(-2 \operatorname{Ric}_{\mu \nu}(g)+g_{\mu \nu}^{\prime \prime}-g_{\mu \lambda}^{\prime} g^{\lambda \sigma} g_{\sigma \nu}^{\prime}+\frac{1}{2} \operatorname{Tr}\left(g^{-1} g^{\prime}\right) g_{\mu \nu}^{\prime}\right. \\
& \left.-(d-1) \frac{1}{z} g_{\mu \nu}^{\prime}-\frac{1}{z} \operatorname{Tr}\left(g^{-1} g^{\prime}\right) g_{\mu \nu}+\frac{2 d}{z^{2}} g_{\mu \nu}\right),
\end{aligned}
$$


where

$$
\operatorname{Ric}(g)_{\mu \nu}=\partial_{\alpha} \Gamma_{\mu \nu}^{\alpha}(g)-\partial_{\nu} \Gamma_{\mu \alpha}^{\alpha}(g)+\Gamma_{\beta \alpha}^{\alpha}(g) \Gamma_{\nu \mu}^{\beta}(g)-\Gamma_{\mu \beta}^{\alpha}(g) \Gamma_{\nu \alpha}^{\beta}(g)
$$

The scalar curvature is given by

$$
\begin{aligned}
R[G] & =G^{M N} R[G]_{M N}=G^{z z} R[G]_{z z}+G^{\mu \nu} R[G]_{\mu \nu} \\
& =z^{2}\left(-\operatorname{Tr} g^{-1} g^{\prime \prime}+\frac{3}{4} \operatorname{Tr} g^{-1} g^{\prime} g^{-1} g^{\prime}+\frac{d}{z} \operatorname{Tr} g^{-1} g^{\prime}-\frac{1}{4}\left(\operatorname{Tr} g^{-1} g^{\prime}\right)^{2}-\frac{d^{2}+d}{z^{2}}+R(g)\right),
\end{aligned}
$$

where $R(g)=g^{\mu \nu} \operatorname{Ric}(g)_{\mu \nu}$.

We expand $\operatorname{Ric}(g)_{\mu \nu}$ with respect to $h_{\mu \nu}$ in (4.2):

$$
\operatorname{Ric}(g)_{\mu \nu}=\operatorname{Ric}^{(1)}(h)_{\mu \nu}+\operatorname{Ric}^{(2)}(h)_{\mu \nu}+\ldots
$$

where the first order of $h_{\mu \nu}, \operatorname{Ric}^{(1)}(h)_{\mu \nu}$, is

$$
\operatorname{Ric}^{(1)}(h)_{\mu \nu}=\frac{1}{2} \partial^{\alpha}\left(\partial_{\mu} h_{\nu \alpha}+\partial_{\nu} h_{\mu \alpha}-\partial_{\alpha} h_{\mu \nu}\right)-\frac{1}{2} \partial_{\mu} \partial_{\nu} h_{\alpha}^{\alpha}
$$

and the second order of $h_{\mu \nu}, \operatorname{Ric}^{(2)}(h)_{\mu \nu}$, is

$$
\begin{aligned}
\operatorname{Ric}^{(2)}(h)_{\mu \nu}= & -\frac{1}{2} \partial_{\alpha}\left\{h^{\alpha \beta}\left(\partial_{\mu} h_{\nu \beta}+\partial_{\nu} h_{\mu \beta}-\partial_{\beta} h_{\mu \nu}\right)\right\} \\
& +\frac{1}{2} \partial_{\nu}\left\{h^{\alpha \beta} \partial_{\mu} h_{\alpha \beta}\right\}+\frac{1}{4} \partial^{\beta} h_{\alpha}^{\alpha}\left(\partial_{\mu} h_{\nu \beta}+\partial_{\nu} h_{\mu \beta}-\partial_{\beta} h_{\mu \nu}\right) \\
& -\frac{1}{4} \delta^{\alpha \gamma} \delta^{\beta \delta}\left(\partial_{\mu} h_{\beta \gamma} \partial_{\mu} h_{\alpha \delta}+\partial_{\beta} h_{\mu \gamma} \partial_{\alpha} h_{\mu \delta}\right. \\
& \left.\quad-\partial_{\beta} h_{\mu \gamma} \partial_{\delta} h_{\mu \alpha}-\partial_{\gamma} h_{\mu \beta} \partial_{\delta} h_{\mu \alpha}+\partial_{\gamma} h_{\mu \beta} \partial_{\delta} h_{\mu \alpha}\right) .
\end{aligned}
$$

Then, we obtain the second order of $h_{\mu \nu}$ in $R(g)$,

$$
\begin{aligned}
R^{(2)}(h)= & \delta^{\mu \nu} \operatorname{Ric}^{(2)}(h)_{\mu \nu}-h^{\mu \nu} \operatorname{Ric}^{(1)}(h)_{\mu \nu} \\
= & -\frac{1}{2} \partial_{\alpha}\left\{h^{\alpha \beta}\left(2 \partial^{\mu} h_{\mu \beta}-\partial_{\beta} h_{\mu}^{\mu}\right)\right\} \\
& +\frac{1}{2} \partial^{\mu}\left\{h^{\alpha \beta} \partial_{\mu} h_{\alpha \beta}\right\}+\frac{1}{4} \partial^{\beta} h_{\alpha}^{\alpha}\left(2 \partial^{\mu} h_{\mu \beta}-\partial_{\beta} h_{\mu}^{\mu}\right) \\
& -\frac{1}{4}\left(2 \partial^{\alpha} h^{\mu \nu} \partial_{\nu} h_{\mu \alpha}-\partial_{\alpha} h^{\mu \nu} \partial_{\alpha} h_{\mu \nu}\right) \\
& -h^{\mu \nu}\left\{\frac{1}{2} \partial^{\alpha}\left(2 \partial_{\mu} h_{\nu \alpha}-\partial_{\alpha} h_{\mu \nu}\right)-\frac{1}{2} \partial_{\mu} \partial_{\nu} h_{\alpha}^{\alpha}\right\} .
\end{aligned}
$$


We expand the Einstein-Hilbert action and the Gibbons-Hawking term up tp $\mathcal{O}\left(h^{2}\right)$ :

$$
\begin{aligned}
S_{\mathrm{EH}}= & \frac{1}{16 \pi G_{N}} \int d^{d+1} x \sqrt{G}(-R[G]+2 \Lambda) \\
= & \frac{1}{16 \pi G_{N}} \int d^{d+1} x z^{-d+1}\left\{-\frac{2 d}{z^{2}}+h^{\prime \prime \mu}{ }_{\mu}-\frac{d}{z} h_{\mu}^{\prime \mu}+\frac{d}{z^{2}} h_{\mu}^{\mu}\right. \\
& -h^{\mu \nu} h^{\prime \prime}{ }_{\mu \nu}-\frac{3}{4} h^{\prime \mu \nu} h_{\mu \nu}^{\prime}+\frac{d}{z} h^{\mu \nu} h_{\mu \nu}^{\prime}-\frac{d}{2 z^{2}} h^{\mu \nu} h_{\mu \nu}+\frac{1}{4} \partial^{\alpha} h^{\mu \nu} \partial_{\alpha} h_{\mu \nu}-\frac{1}{2} \partial^{\alpha} h^{\mu \nu} \partial_{\mu} h_{\mu \alpha} \\
& \left.+\frac{1}{2} h_{\mu}^{\mu} h_{\nu}^{\prime \prime \nu}+\frac{1}{4}\left(h^{\prime \mu}\right)^{2}-\frac{d}{2 z} h_{\mu}^{\nu} h^{\prime \nu}+\frac{d}{4 z^{2}}\left(h_{\mu}^{\mu}\right)^{2}+\frac{1}{2} \partial_{\nu} h^{\mu \nu} \partial_{\mu} h_{\alpha}^{\alpha}-\frac{1}{4} \partial^{\alpha} h_{\mu}^{\mu} \partial_{\alpha} h_{\nu}^{\nu}\right\} \\
S_{\mathrm{GH}}= & -\frac{1}{16 \pi G_{N}} \int_{z=\epsilon} d^{d} x \sqrt{\gamma}(2 K+\lambda) \\
= & \frac{1}{16 \pi G_{N}} \int_{z=\epsilon} d^{d} x z^{-d}\left\{-2-h_{\mu}^{\mu}-\frac{1}{4}\left(h_{\mu}^{\mu}\right)^{2}+\frac{1}{2} h^{\mu \nu} h_{\mu \nu}-h^{\mu \nu} h_{\mu \nu}^{\prime}+h^{\prime \mu}+\frac{1}{2} h_{\mu}^{\mu} h_{\nu}^{\prime \nu}\right\}
\end{aligned}
$$

By using the Einstein equations to the first order of $h_{\mu \nu},(6.6),(6.7),(6.8)$ and (6.9), the Einstein-Hilbert action $S_{\mathrm{EH}}$ is reduced to

$$
S_{\mathrm{EH}, \text { on-shell }}=\frac{1}{16 \pi G_{N}} \int d^{d+1} x \frac{d}{d z}\left\{-\frac{3}{4} z^{-d+1} h^{\mu \nu} h_{\mu \nu}^{\prime}+\frac{1}{2} z^{-d} h^{\mu \nu} h_{\mu \nu}-2 z^{-d}\right\}
$$

and the Gibbons-Hawking term $S_{\mathrm{GH}}$ is reduced to

$$
S_{\mathrm{GH}, \mathrm{on}-\mathrm{shell}}=\frac{1}{16 \pi G_{N}} \int_{z=\epsilon} d^{d} x z^{-d}\left\{-z h^{\mu \nu} h_{\mu \nu}^{\prime}+\frac{1}{2} h_{(1)}^{\mu \nu} h_{(1) \mu \nu}-2\right\}
$$

Thus, using (A.20) and (A.21), we obtain the on-shell action (6.12) on the boundary specified by $z=\epsilon$.

Open Access. This article is distributed under the terms of the Creative Commons Attribution License (CC-BY 4.0), which permits any use, distribution and reproduction in any medium, provided the original author(s) and source are credited.

\section{References}

[1] J.M. Maldacena, The Large $N$ limit of superconformal field theories and supergravity, Int. J. Theor. Phys. 38 (1999) 1113 [hep-th/9711200] [INSPIRE].

[2] S.S. Gubser, I.R. Klebanov and A.M. Polyakov, Gauge theory correlators from noncritical string theory, Phys. Lett. B 428 (1998) 105 [hep-th/9802109] [INSPIRE].

[3] E. Witten, Anti-de Sitter space and holography, Adv. Theor. Math. Phys. 2 (1998) 253 [hep-th/9802150] [INSPIRE].

[4] L. Susskind and E. Witten, The Holographic bound in anti-de Sitter space, hep-th/9805114 [INSPIRE].

[5] J. de Boer, E.P. Verlinde and H.L. Verlinde, On the holographic renormalization group, JHEP 08 (2000) 003 [hep-th/9912012] [INSPIRE]. 
[6] S. Ryu and T. Takayanagi, Holographic derivation of entanglement entropy from AdS/CFT, Phys. Rev. Lett. 96 (2006) 181602 [hep-th/0603001] [INSPIRE].

[7] M. Miyaji, T. Numasawa, N. Shiba, T. Takayanagi and K. Watanabe, Distance between Quantum States and Gauge-Gravity Duality, Phys. Rev. Lett. 115 (2015) 261602 [arXiv: 1507.07555] [INSPIRE].

[8] D. Bak, Information metric and Euclidean Janus correspondence, Phys. Lett. B 756 (2016) 200 [arXiv: 1512.04735] [INSPIRE].

[9] A. Trivella, Holographic Computations of the Quantum Information Metric, Class. Quant. Grav. 34 (2017) 105003 [arXiv:1607.06519] [INSPIRE].

[10] C.-B. Chen, W.-C. Gan, F.-W. Shu and B. Xiong, Quantum information metric of conical defect, Phys. Rev. D 98 (2018) 046008 [arXiv: 1804.08358] [InSPIRE].

[11] S. Karar, R. Mishra and S. Gangopadhyay, Holographic complexity of boosted black brane and Fisher information, Phys. Rev. D 100 (2019) 026006 [arXiv:1904.13090] [INSPIRE].

[12] M. Nozaki, S. Ryu and T. Takayanagi, Holographic Geometry of Entanglement Renormalization in Quantum Field Theories, JHEP 10 (2012) 193 [arXiv:1208.3469] [INSPIRE].

[13] N. Lashkari and M. Van Raamsdonk, Canonical Energy is Quantum Fisher Information, JHEP 04 (2016) 153 [arXiv: 1508.00897] [INSPIRE].

[14] S. Aoki and S. Yokoyama, Flow equation, conformal symmetry and anti-de Sitter geometry, PTEP 2018 (2018) 031B01 [arXiv: 1707.03982] [INSPIRE].

[15] A. May and E. Hijano, The holographic entropy zoo, JHEP 10 (2018) 036 [arXiv: 1806.06077] [INSPIRE].

[16] H. Liu and A.A. Tseytlin, $D=4$ superYang-Mills, $D=5$ gauged supergravity and $D=4$ conformal supergravity, Nucl. Phys. B 533 (1998) 88 [hep-th/9804083] [INSPIRE]. 